\title{
Separation of Sodiated Isobaric Disaccharides and Trisaccharides Using Electrospray Ionization-Atmospheric Pressure Ion Mobility-Time of Flight Mass Spectrometry
}

\author{
Brian H. Clowers, ${ }^{*}$ Prabha Dwivedi, Wes E. Steiner, \\ and Herbert H. Hill, Jr. \\ Department of Chemistry, Washington State University, Pullman, Washington, USA \\ Brad Bendiak \\ Cell and Developmental Biology, University of Colorado Health Sciences Center, Denver, Colorado, USA
}

\begin{abstract}
A series of isobaric disaccharide-alditols, four derived from O-linked glycoproteins, and select trisaccharides were rapidly resolved using tandem high resolution atmospheric pressure ion-mobility time-of-flight mass spectrometry. Electrospray ionization was used to create the gas-phase sodium adducts of each carbohydrate. Using this technique it was possible to separate up to three isobaric disaccharide alditols and three trisaccharides in the gas phase. Reduced mobility values and experimentally determined ion-neutral cross sections are reported for each sodium-carbohydrate complex. These studies demonstrated that ion mobility separations at atmospheric pressure can provide a high-resolution dimension for analysis of carbohydrate ions that is complementary to traditional mass spectral $(\mathrm{m} / \mathrm{z})$ ion analysis. Combining these independent principles for separation of ions provides a powerful new bioanalytical tool for the identification of isomeric carbohydrates. (J Am Soc Mass Spectrom 2005, 16, 660-669) (c 2005 American Society for Mass Spectrometry
\end{abstract}

$\mathrm{I}$ ndividually, carbohydrates are a primary source of energy in numerous biological systems; however, when attached to cellular lipids and proteins, carbohydrates play pivotal roles in cellular recognition, signaling, receptor binding, and consequently immune responses [1-4]. These roles are often the result of oligosaccharides linked to proteins as post-translational modifications (PTMs). Carbohydrates that fall under this category are primarily linked to proteins through an $\mathrm{O}$ - or N-linked $\mathrm{N}$-acetyl hexosamine, primarily $\mathrm{N}$ acetyl glucosamine (GlcNAc) or $\mathrm{N}$-acetyl galactosamine (GalNAc) [5]. Given the wide range and importance of carbohydrates in biological systems, their unambiguous identification is of utmost importance [6-8].

In order to fully characterize oligosaccharides derived from biological sources, a number distinct of challenges must be over come. These issues arise primarily from the existence of biological oligosaccharides as sets of isomers. A number of approaches to address isomeric carbohydrate structures using mass spectrometry have been reported. These methods include perio-

Published online March 10, 2005

Address reprint requests to Professor H. H. Hill, Department of Chemistry, Washington State University, 100 Dairy Road, Pullman, WA 99164, USA. E-mail: hhhill@wsu.edu

* Also at the Center for Multiphase Environmental Research, Washington State University, Pullman, WA 99164, USA. date oxidation/borohydride reduction, followed by hydroxyl methylation or peracetylation $[9,10]$, derivatization of monosaccharides or short oligosaccharides with amines such as diethylenetriamine at the reducing end followed by metal complexation [11, 12], or prediction of possible fragmentation pathways after permethylation [13]. Although modern mass spectrometry is an exquisite tool in itself for the separation of molecules having different $m / z$ values, it cannot rule out the possibility that mass spectra derived from selected precursor ions are not derived from an isobaric mixture. And to complicate matters even further, it is entirely possible that the fragment ions themselves are isobars. On a fundamental level, the stereochemistry of monosaccharides, as product ions derived from a larger molecule, cannot be unambiguously established from a fragmentation pattern. Aldohexoses, for example, come in sixteen different stereochemical variants, and fragmentation data that would uniquely differentiate each of them has yet to be convincingly furnished, particularly where they are derived as product ions from larger molecules. To address the stereochemical blindness of mass spectrometry, product ions must first be separated based upon a physical principle that is not dependent upon $\mathrm{m} / \mathrm{z}$ prior to fragmentation.

Traditional chromatographic methods, most notably liquid and gas, have been used to provide an additional 
dimension of information prior to mass analysis; however, these techniques rely upon differential partition coefficients which in many cases may be insufficient to reproducibly differentiate between isomeric carbohydrates [8, 14]. Ion mobility spectrometry (IMS) possesses the unique ability to separate gas-phase ions based upon their size to charge ratio and provides a fresh alternative to traditional modes of separating isomeric and enantiomeric species. Since its introduction as a new dimension for gas chromatographic and mass spectrometric applications, IMS has grown over the past quarter of a century to become an important analytical separation technique. Compared to traditional liquid and gas-phase separation techniques, IMS is temporally fast (in the millisecond time scale), mechanically robust, and sensitive to a wide range of chemical systems [15-20]. New instrumental developments and configurations have strikingly improved ion mobility as a separation technique allowing for resolving powers to exceed those of liquid chromatography (LC) and rival those of gas chromatography (GC) [2022]. Combining this post-ionization gas-phase separation method with mass spectrometry has recently demonstrated the technique's utility as a rapid means to distinguish between isomeric forms of select compounds [23]. With the advent of electrospray ionization (ESI), matrix assisted laser desorption ionization (MALDI), and controlled enzymatic forms of protein digestion, IMS has been applied to the separation of peptide isomers [24, 25].

Individually carbohydrates have been investigated previously with IMS, however, these data were obtained with relatively low resolution IMS instrumentation [26-28]. These studies indicated that small differences in drift times of individual precursor ions could be obtained both in the positive and negative ion modes, although full resolution of isomers from mixtures was not demonstrated. Using a different approach, Leavell et al. combined computational methods and ion mobility mass spectrometry (IMMS) to examine the gas-phase ion structure of diethylenetriamine-derivatized sugars as zinc-ligand-hexose diastereomers [29]. Their studies were performed with derivatized reducing sugars hence multiple peaks were observed for individual sugars representing different configurations of complexes. While the above studies provided unique insights into the behavior of complexes of metal ions with carbohydrates in the gas phase, they did not demonstrate the utility of IMS as a separation technique prior to mass analysis; the carbohydrates chosen for analysis were examined individually and not in a mixture. To our knowledge, this is the first report of the complete physical resolution of isomeric carbohydrate structures from mixtures using atmospheric pressure high resolution ion mobility time-of-flight mass spectrometry.

Gas phase separations of carbohydrates have been reported using field asymmetric ion mobility spectrometry (FAIMS) [30]. These studies demonstrate the tech- nique's unique ability to provide information regarding carbohydrate systems. However, the time frame of separation using FAIMS coupled with quadrupole mass spectrometry is rather long $(\sim 1 \mathrm{~h})$ by comparison to IMS ( $\sim 5 \mathrm{~min})$. Further issues remain regarding signal to noise ratio (SNR) and the appearance of multiple peaks attributable to cluster dissociation. Nonetheless, FAIMS does permit studies of complex fragile ion clusters to be performed which is less directly applicable to conformational structural elucidation, but is important in its own right.

\section{Experimental}

\section{Sample Preparation}

The disaccharides examined in this study were a combination of the monosaccharide units $N$-acetyl-D-galactosamine (GalNAc), N-acetyl-D-glucosamine (GlcNAc), D-glucose (Glc), and D-galactose (Gal). Dissacharides were as follows: melibiitol ( $\alpha$-D-Gal-(1-6)-D-Glc-ol), cellobiitol ( $\beta$-D-Glc-(1-4)-D-Glc-ol, $\alpha$-D-GalNAc-(1-6)D-GalNAc-ol, $\quad \alpha$-D-GalNAc-(1-3)-D-GalNAc-ol, $\quad \beta$ D-GlcNAc-(1-6)-D-GalNAc-ol, $\quad \beta$-D-GlcNAc-(1-3)D-GalNAc-ol, and $\beta$-D-GlcNAc-(1-4)-D-GlcNAc-ol. According the procedure outlined by Martensson et al., all of the disaccharide-alditols used in this study, aside from $\beta$-D-GlcNAc-(1-4)-D-GlcNAc-ol, cellobiitol, and melibiitol, were isolated from bovine submaxillary mucin (BSM) [31]. Briefly, the neutral and negativelycharged saccharides released from the protein by alkaline borohydride treatment were separated by ion exchange chromatography. Pools were further fractionated by multiple HPLC columns and analyzed after purification to homogeneity by NMR and MS/MS. Cellobiose, melibiose, and $\beta$-D-GlcNAc-(1-4)-D-GlcNAc were purchased from Sigma-Aldrich (St. Louis, MO) and reduced with sodium borohydride to generate the sugar-alditols.

Melezitose ( $\alpha$-D-Glc-(1-3)- $\beta$-D-Fru-(2-1)- $\alpha$-D-Glc), raffinose ( $\alpha$-D-Gal-(1-6)- $\alpha$-D-Glc-(1-2)- $\beta$-D-Fru), and isomaltotriose ( $\alpha$-D-Glc-(1-6)- $\alpha$-D-Glc-(1-6)- $\alpha$-D-Glc) were purchased from Sigma-Aldrich (St. Louis, MO) and used without further purification.

Purified samples $(\sim 10 \mu \mathrm{mol})$ of each disaccharide were dissolved in $1 \mathrm{~mL}$ of $18.1 \mathrm{M} \Omega$ water. From these 10 $\mathrm{mM}$ stock solutions further dilutions to $100 \mathrm{pmol} / \mu \mathrm{L}$ in 90:10 (water:methanol) were made prior to analysis. For melezitose, raffinose, and isomaltotriose, each was analyzed at a concentration of $50 \mathrm{pmol} / \mu \mathrm{L}$ in 90:10 (water:methanol). To ensure sodium adduction, 25 $\mathrm{pmol} / \mu \mathrm{L}$ of sodium chloride was added to each trisaccharide sample before analysis.

\section{Electrospray Ionization-Atmospheric Pressure Ion Mobility-Time-of-Flight Mass Spectrometry}

Separation experiments were conducted using the electrospray ionization atmospheric pressure ion mobility 


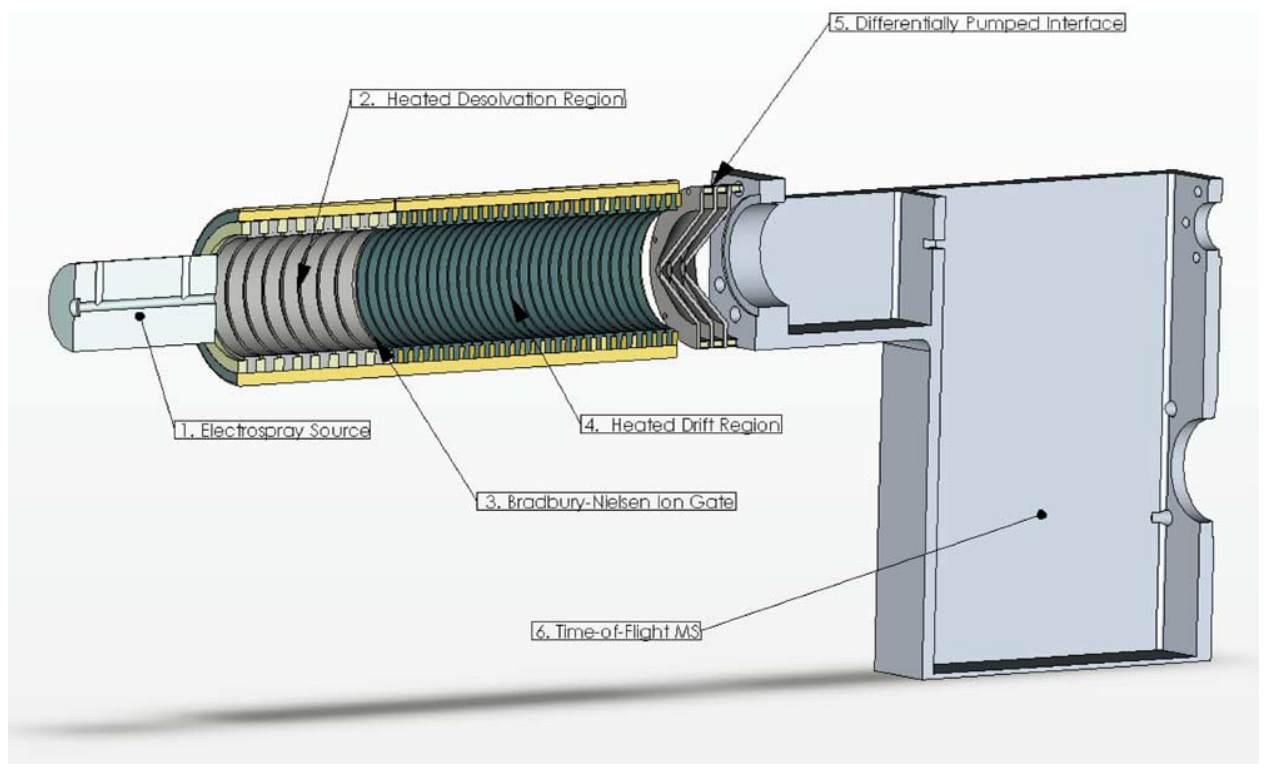

Figure 1. Schematic of the electrospray ionization atmospheric pressure ion mobility time-of-flight mass spectrometer used for the separation and identification of isobaric carbohydrates. This instrument is comprised of seven primary units: (1) electrospray ionization source; (2) heated atmospheric pressure desolvation region; (3) Bradbury-Nielsen ion gate; (4) counter-flow atmospheric pressure drift region; (5) differentially pumped interface; (6) time-of-flight mass analyzer; (7) PC based data acquisition system (not shown).

time-of-flight mass spectrometer (ESI-AP-IM-TOFMS), Figure 1, constructed at Washington State University. This instrument was comprised of the following units: (1) ESI source; (2) Heated AP desolvation region; (3) Bradbury-Nielsen ion gate; (4) counter-flow AP drift region; (5) differentially pumped interface; (6) TOF $\mathrm{m} / \mathrm{z}$ analyzer; and (7) data acquisition system. A detailed description of the fundamental operating principles and parameters may be found in reference [32], hence only a brief outline is presented. Samples of each carbohydrate were infused at $3 \mu \mathrm{L} / \mathrm{min}$ through the electrospray unit maintained at $13 \mathrm{kV}(\sim 3.0 \mathrm{kV}$ greater than the potential applied across the ion mobility tube). Ionized sample molecules were desolvated prior to ion gating using a counter-current flow $(\sim 1 \mathrm{~L} / \mathrm{min})$ of heated nitrogen drift gas held at $200{ }^{\circ} \mathrm{C}$. With a larger center of mass and collision cross section, nitrogen allows for longer ion drift times, and consequently higher resolving powers, when compared to drift gases such as helium [27, 33]. The ambient pressure in Pullman, WA was approximately 700 torr. The mobility separation sequence was initiated by the pulsing of a Bradbury-Nielsen ion gate operating at $\sim 35 \mathrm{~Hz}$. Once in the $18.1 \mathrm{~cm}$ drift region, the ions were directed by a uniform electric field of $420 \mathrm{~V} / \mathrm{cm}$ towards the $200 \mu \mathrm{m}$ aperture of the orthogonal TOF mass analyzer maintained at $10^{-6}$ torr. To enhance signal to noise ratio (SNR) for each measurement, signal averaging was employed with an average analysis time of $\sim 10 \mathrm{~min}$. It should be noted that, given the disparate time scales in which ion mobility and TOF instruments operated, multiple mass spectra were acquired during the course of a single ion mobility pulse. Ion drift times at atmospheric pressure occur on the millisecond time scale, whereas, ion flight times in the TOF $\mathrm{m} / \mathrm{z}$ analyzer are on the microsecond scale.

\section{Reduced Mobility Constants, Resolving Power, and Experimentally Determined Cross Sections}

In the presence of a weak homogenous electric field, ions possessing different collision cross-sections temporally separate based on the frequency of ion-molecule interactions. The mobility of an ion $(K)$ is defined as its velocity $\left(v_{d}\right)$ divided by the applied electric field $(E)$ and can be determined from experimental parameters as follows:

$$
\mathrm{K}=\frac{\mathrm{V}_{\mathrm{d}}}{\mathrm{E}}=\frac{\mathrm{L}^{2}}{\mathrm{t}_{\mathrm{d}} \mathrm{V}}
$$

where $L$ is the length of the drift region $(\mathrm{cm}), t_{d}$, the drift time (seconds) and , $V$, the potential applied to the drift region (volts). The drift tube temperature $(T)$ typically varies from ambient temperature to $800 \mathrm{~K}$ and operating pressures from 1 torr up to atmospheric pressure [34]. As the mobility experiment may operate under a wide range of operating conditions the mobility of an ion must be normalized $\left(\mathrm{K}_{\mathrm{o}}\right)$ for drift gas pressure $(P)$ and temperature $(\mathrm{T})$ to allow for comparisons between experimental systems. The resulting reduced mobility value is determined as shown below: 


$$
\mathrm{K}_{\mathrm{o}}=\mathrm{K} \frac{273.15}{\mathrm{~T}} \frac{\mathrm{P}}{760}
$$

The efficiency of separation or resolving power of ion mobility spectrometry has traditionally been defined as the drift time $\left(t_{d}\right)$ divided by the peak width at half height $\left(w_{1 / 2}\right)$ [35].

$$
R_{p}=\frac{t_{d}}{W_{1 / 2}}
$$

It should also be noted that while ion mobility resolving power is a measure of separation efficiency this term does not indicate the degree to which two different peaks are separated. This parameter is defined as the resolution (R) of the separation and is directly analogous to the term defined by the chromatographic community where baseline resolution is achieved with an $R$ value of 1.5. This value is the result of the difference in drift times $\left(\Delta t_{d}\right)$ divided by the average baseline peak width $\left(\mathrm{w}_{\text {base }}\right)$ [36].

$$
\mathrm{R}=\frac{\Delta \mathrm{t}_{\mathrm{d}}}{\overline{\mathrm{W}}_{\text {base }}}
$$

While ion mobility provides the means to separate gas-phase ion populations it also provides the means to measure gas-phase ion-neutral collision cross sections. The ion-neutral collision cross section $(\Omega)$ can be related to the mobility by the following equation under low field conditions:

$$
\Omega=\left(\frac{3}{16 \mathrm{~N}}\right)\left(\frac{2 \pi}{\mu \mathrm{kT}}\right)^{1 / 2}\left(\frac{\mathrm{ze}}{\mathrm{K}_{\mathrm{o}}}\right)
$$

Where $z$ is the number of the charges on the ion; $e$ the charge of one proton; $N$ the number density of the drift gas at standard conditions, $\mu=[\mathrm{mM} /(m+M)]$ the reduced mass of an ion $(m)$ and the neutral drift gas $(M)$, and $k$, Boltzmann's constant [33, 37]. Using a variety of computational methods it is possible to gain insight into the structure of gas-phase ions by correlating experimentally determined collision cross sections with theoretically derived structures [29, 33, 34, 38-40].

\section{Results and Discussion}

\section{$N$-Acetyl Hexosamine Disaccharide-Alditol Separation}

The combination of atmospheric pressure ion mobility with time-of-flight mass spectrometry requires an accurate means of measuring ion drift time under atmospheric and vacuum conditions. As discussed previously, the orders of magnitude difference in time scales between ion mobility and TOF separation times allows numerous mass spectra to be acquired during the course of a single ion mobility pulse. Drift times for ion populations traversing the ion mobility tube are on the order of 10s of milliseconds; whereas, drift times within the TOF system are a few microseconds. This large difference allows the time spent by an ion population in the mass spectrometer to be neglected with respect to the overall ion mobility drift time as it is within the error of the mobility measurement. The union of these two time-of-flight techniques allows data to be acquired and displayed in a two dimensional matrix which is best represented in a contour plot, as shown in Figure 2. This spectrum is the ESI-AP-IM-TOFMS contour plot of sodium adduct $[\mathrm{M}+\mathrm{Na}]^{+}$of cellobiitol ( $\beta$-D-Glc-(1-4)D-Glc-ol). The drift time of the ion populations in microseconds is shown on the y-axis and the corresponding $\mathrm{m} / \mathrm{z}$ value for each population shown on the $\mathrm{x}$-axis located above the contour plot.

Because excessive fragmentation may complicate spectral interpretation it was our expressed intent not to induce fragmentation of ions as they pass through the differentially pumped pressure region. Collision induced dissociation may be initiated using AP-IM-TOFMS by altering the potential gradient between the atmospheric pressure aperture and the skimmer prior to entering the TOF mass analyzer. Due to the fact that an ion's flight time within the TOFMS is $\sim 4$ orders of magnitude faster than its corresponding ion mobility drift time at atmospheric pressure, both the fragment and parent ions possess statistically identical drift times. A more detailed description of ion fragmentation behavior post ion mobility separation may be found in references [32] and [41].

During the course of our experiments, we were unable to detect fragmentation of the parent ions as they traversed the differentially pumped vacuum region. Further, our experimental evidence suggests the ions studied were stable throughout the ion mobilityTOF experiment, as fragmentation within the drift tube would produce broad, poorly defined peaks and disrupt the separation characteristics of the experiment. It should also be noted that dimers for each disaccharide were not observed, either as a standard or in a mixture. Given the absence of detectable fragmentation and dimer formation, we have chosen a zoomed view in both the mass and mobility dimensions to best illustrate the features of each contour separation.

When analyzed individually, all of the disaccharides used in this study produced a spectrum similar to the data displayed in Figure 2. As outlined in the experimental procedure, the structures of each disaccharide were reduced. This prevented contributions to spectra from different configurations that may have existed in solution. Additionally, chemical reduction also avoided any issues with potential interconversions between $\alpha$ and $\beta$ or pyranose and furanose ring forms during the actual experiment. While no definitive evidence currently exists to suggest the rapid interconversion of the reducing sugar of oligosaccharides in the gas phase, such an occurrence during the course of an ion mobility 


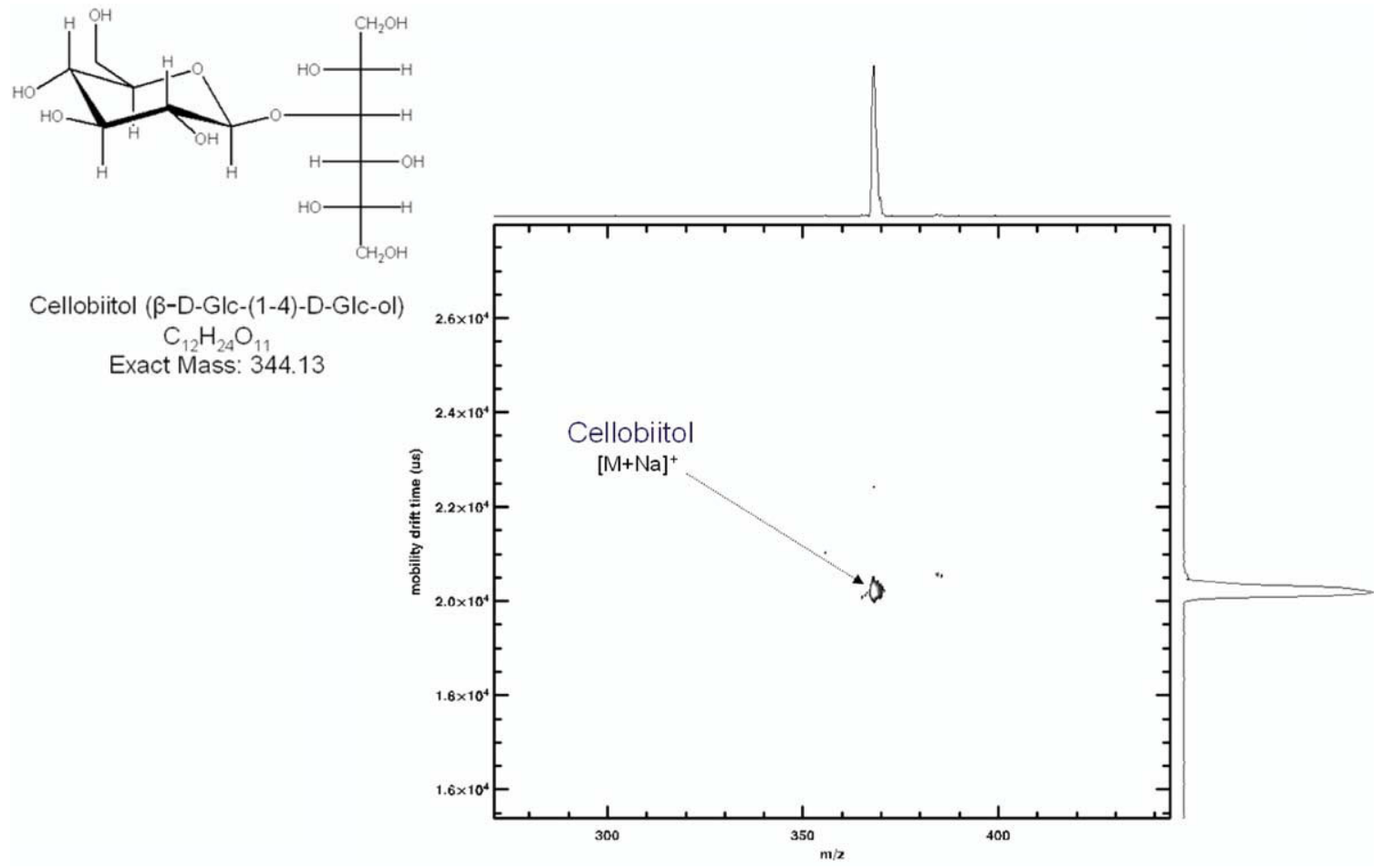

Figure 2. An ion-mobility/mass spectral two-dimensional contour plot of the sodiated adduct [M + $\mathrm{Na}]^{+}$(367.05) of cellobiitol ( $\beta$-D-Glc-(1-4)-D-Glc-ol). Shown in the panel is the correlation between the ion mobility drift time (in this case $20.2 \mathrm{~ms}$ ) and the $\mathrm{m} / \mathrm{z}$ (367.05). On the top is shown a trace of the TOF-determined $\mathrm{m} / \mathrm{z}$ and along the side is shown the intensity of the ion signal with different ion mobility drift times. For this particular disaccharide-alditol concentration $(100 \mathrm{pmol} / \mu \mathrm{L})$ the SNR was 167.

experiment could result in peak broadening and a loss of resolving power.

It should also be noted that despite the best efforts to eliminate sodium contamination from the experimental procedure, the carbohydrate ions examined were almost exclusively found to exist as sodium adducts. Clearly, each disaccharide-alditol binds effectively to trace amounts of sodium present in the sample; however, the mechanism of coordination is believed to be somewhat different between isomers $[29,34]$. It is this potential difference in sodium coordination that is hypothesized to give rise to the small changes in ion-neutral collision cross sections and the consequent isomeric separation. Previous research on the thermochemistry and structure of sodium-disaccharide adducts has indicated that the binding is multidentate in nature [42]; however, no collision cross section measurements have been made for disaccharides containing $\mathrm{N}$-acetylhexosamine residues. The reduced mobility and experimentally determined collision cross section for each sodium disaccharide-alditol adduct are reported in Table 1.

Table 1. Reduced mobilities and ion-neutral collision cross sections determined for select carbohydrates

\begin{tabular}{|c|c|c|c|c|c|c|c|c|}
\hline Carbohydrate & Adduct & M.W. & Drift time (ms) & FWHM & $\mathrm{K}_{\mathrm{o}}$ & $\mathrm{K}_{\mathrm{ORSD}}$ & $\Omega^{a}$ & $\Omega_{\mathrm{RSD}}$ \\
\hline$\alpha$-D-GaINAc-(1-6)-D-GaINAc-ol ${ }^{\mathrm{b}, \mathrm{c}}$ & $\mathrm{Na}$ & 449.18 & 22.87 & 0.25 & 1.01 & 0.76 & 164.28 & 0.79 \\
\hline$\alpha$-D-GaINAc-(1-3)-D-GaINAc-ol ${ }^{\mathrm{b}, \mathrm{c}}$ & $\mathrm{Na}$ & 449.18 & 22.41 & 0.23 & 1.03 & 0.73 & 160.98 & 0.78 \\
\hline$\beta$-D-GIcNAc-(1-6)-D-GaINAc-ol ${ }^{\mathrm{b}, \mathrm{c}}$ & $\mathrm{Na}$ & 449.18 & 23.49 & 0.27 & 0.98 & 0.80 & 169.70 & 0.81 \\
\hline$\beta$-D-GIcNAc-(1-3)-D-GalNAc-ol ${ }^{\mathrm{b}, \mathrm{c}}$ & $\mathrm{Na}$ & 449.18 & 22.63 & 0.21 & 1.01 & 0.72 & 163.49 & 0.76 \\
\hline$\beta$-D-GIcNAc-(1-4)-D-GIcNAc-ol & $\mathrm{Na}$ & 449.18 & 22.90 & 0.21 & 1.00 & 0.72 & 165.44 & 0.76 \\
\hline Melibiitol & $\mathrm{Na}$ & 367.05 & 20.15 & 0.20 & 1.14 & 0.65 & 146.52 & 0.77 \\
\hline Cellobiitol & $\mathrm{Na}$ & 367.05 & 20.09 & 0.21 & 1.14 & 0.67 & 146.09 & 0.79 \\
\hline Melezitose & $\mathrm{Na}$ & 527.16 & 24.13 & 0.28 & 0.95 & 0.76 & 173.57 & 0.75 \\
\hline Raffinose & $\mathrm{Na}$ & 527.16 & 25.60 & 0.26 & 0.91 & 0.78 & 180.91 & 0.74 \\
\hline Isomaltotriose & $\mathrm{Na}$ & 527.16 & 25.15 & 0.28 & 0.90 & 0.79 & 184.14 & 0.74 \\
\hline
\end{tabular}

alon-Neutral Cross Section $\left(\AA^{2}\right)$ determined using the experimental parameters outlined the section entitled "Electrospray lonization-Atmospheric Pressure lon Mobility-Time-of-Flight Mass Spectrometry."

besignates disaccharides isolated from bovine submaxillary mucin [31].

'Denotes data acquired at 701 Torr, all other data were acquired at 697 Torr.

The relative standard deviations (RSD) of the reduced mobility values $\left(\mathrm{K}_{\mathrm{o}}\right)$ and ion-neutral cross sections $(\Omega)$ are expressed as a percent. 


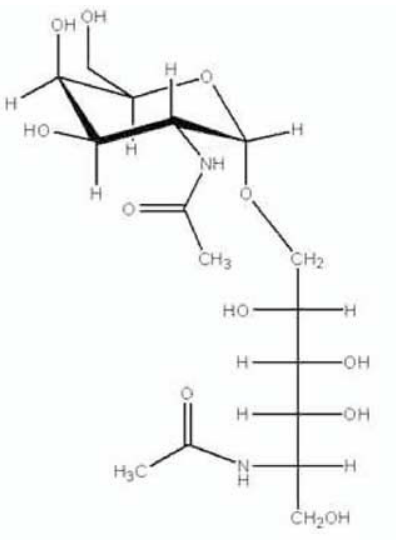

a-D-GalNAc-(1-6)-D-GalNAc-ol

$$
\mathrm{C}_{16} \mathrm{H}_{30} \mathrm{~N}_{2} \mathrm{O}_{11}
$$

Exact Mass: 426.18

a1-6
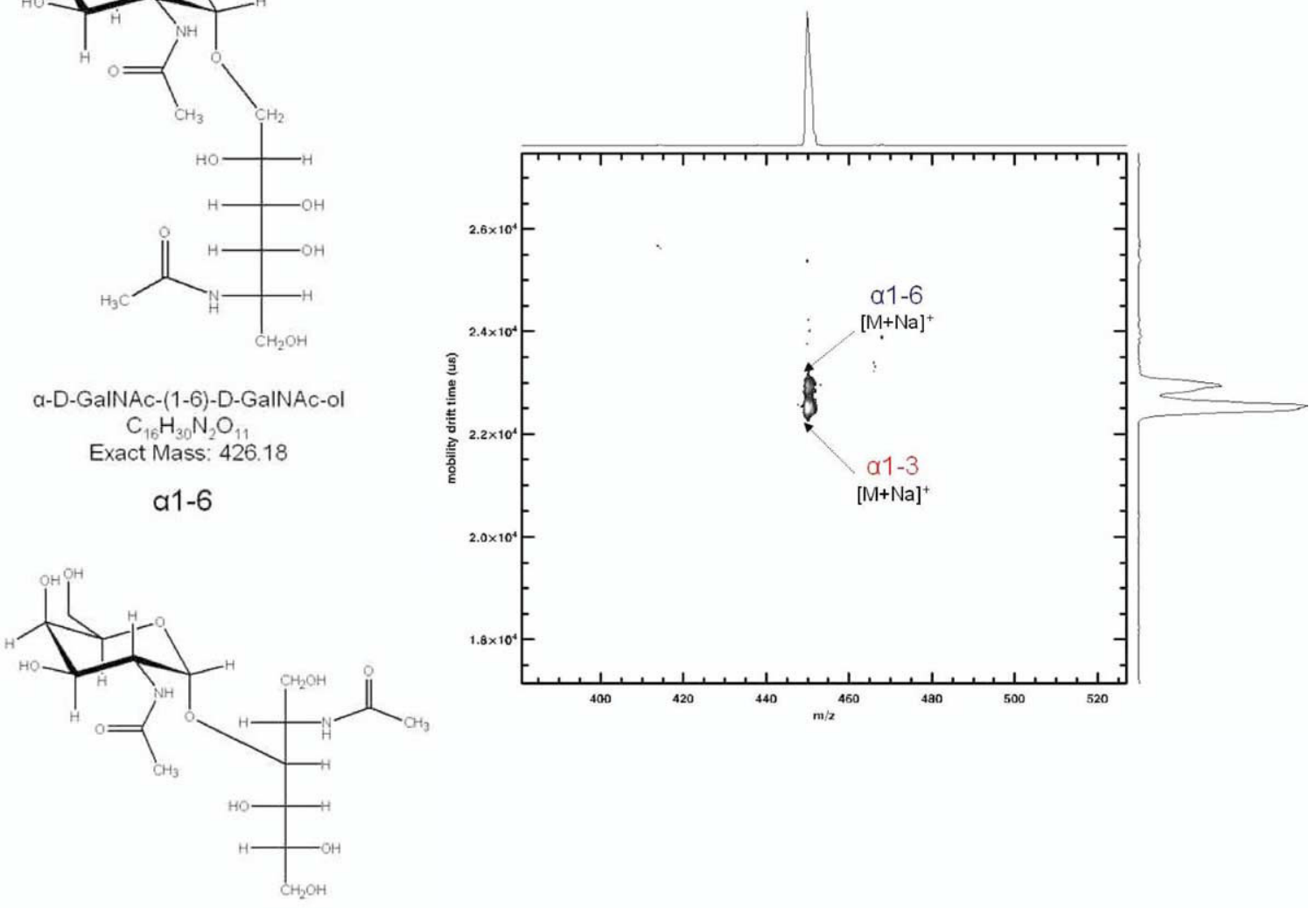

a-D-GalNAc-(1-3)-D-GalNAC-ol
\[ \mathrm{C}_{16} \mathrm{H}_{30} \mathrm{~N}_{2} \mathrm{O}_{11} \]

Exact Mass: 426.18

a1-3

Figure 3. Separation of two isobaric disaccharide-alditols by AP-IM-TOF-MS. Shown in the panel is the contour plot of $\mathrm{m} / \mathrm{z}$ versus ion mobility drift time. Both disaccharide alditols, isolated from bovine submaxillary mucin ( $\alpha$-D-GalNAc-(1-6)-D-GalNAc-ol and $\alpha$-D-GalNAc-(1-3)-D-GalNAc-ol), are exact compositional isomers, and were almost completely separated as their $[\mathrm{M}+\mathrm{Na}]^{+}$ions. Traces along the vertical and horizontal axes illustrate intensities of the ions with $\mathrm{m} / \mathrm{z}$ and ion mobility drift times, respectively. The SNR ratios for this equimolar (100 pmol/ $\mu \mathrm{L})$ of $\alpha-(1-6)$ and $\alpha-(1-3)$, were 77 and 152, respectively.

Prior to acquiring the data pertaining to an isomeric mixture, as shown in Figure 3, the reduced mobility constants for the individual disaccharides were determined (spectra not shown). Figure 3 represents the partial separation of the $[\mathrm{M}+\mathrm{Na}]^{+}$ions of the reduced disaccharide linkage isomers $\alpha$-D-GalNAc-(1-6)-DGalNAc-ol and $\alpha$-D-GalNAc-(1-3)-D-GalNAc-ol using AP-IM-TOF-MS. The mass spectrum contains a singular peak at $m / z 449$, whereas, the mobility spectrum shown on the $y$-axis contains two partially separated peaks. The contour corresponding to the ion mobility peak with the largest intensity is located at $22.41 \mathrm{~ms}$ and corresponds to $\alpha$-D-GalNAc-(1-3)-D-GalNAc-ol. The second and less intense contour corresponding to $\alpha$-DGalNAc-(1-6)-D-GalNAc-ol is located at $22.87 \mathrm{~ms}$. In chromatographic terms Figure 3 contains two peaks separated with a resolution of 1.12 . While not baseline, this resolution is sufficient to clearly identify two distinct peaks within a spectrum. The distinction should again be made between the determined resolution value and the concept of resolving power (eq 3) which is a measure of separation efficiency. On average the ion mobility peaks shown in Figure 3 possessed a resolving power of 96.4 .

In addition to functioning as a gas-phase ion separation technique, ion mobility may assist in providing more information regarding the structure and or conformations of ions. Using eq 5 and the experimentally obtained ion drift times from the data set shown in Figure 3 the ion-neutral cross sections for $\alpha$-D-GalNAc(1-6)-D-GalNAc-ol and $\alpha$-D-GalNAc-(1-3)-D-GalNAc-ol were found to be 164 and $161 \AA^{2}$, respectively.

To further demonstrate the ability of AP-IM-TOF-MS to distinguish between isobaric forms of disaccharides, 


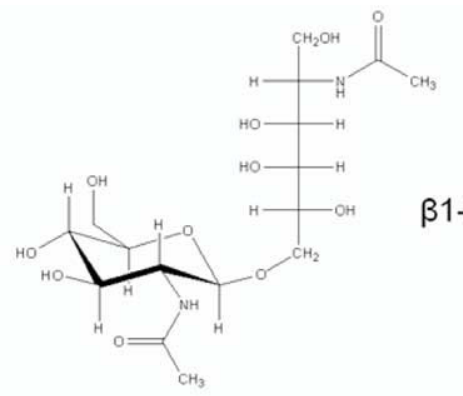

$\beta 1-6$

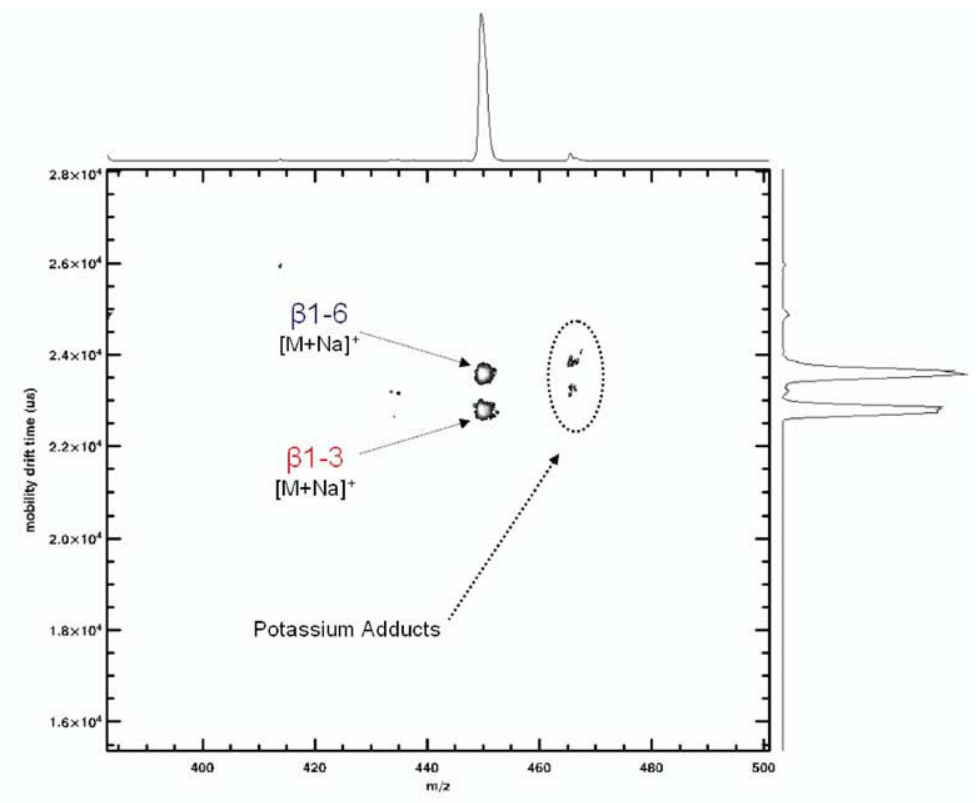

$\beta$-D-GIcNAc-(1-3)-D-GalNAc-ol
\[ \mathrm{C}_{16} \mathrm{H}_{30} \mathrm{~N}_{2} \mathrm{O}_{11} \]

Exact Mass: 426.18

Figure 4. Baseline separation of two linkage isomers of disaccharide-alditols isolated from bovine submaxillary mucin by AP-IM-TOF-MS ( $\beta$-D-GlcNAc-(1-6)-D-GalNAc-ol and $\beta$-D-GlcNAc-(1-3)-D-GalNAcol). Illustrated in the panel is the contour plot of $\mathrm{m} / \mathrm{z}$ versus ion mobility drift time. Traces along vertical and horizontal axes illustrate intensities of the ions with $\mathrm{m} / \mathrm{z}$ and ion mobility drift times, respectively. The primary ion contours correspond to the sodium adducts of the individual disaccharides, whereas, the low intensity contours enclosed by the dotted ellipse are potassium adducts of the same compound. For this equimolar mixture $(100 \mathrm{pmol} / \mu \mathrm{L})$ the average SNR ratio for the dominant peaks was 345 .

Figure 4 displays the baseline separation of $\beta$-DGlcNAc-(1-6)-D-GalNAc-ol and $\beta$-D-GlcNAc-(1-3)-DGalNAc-ol. Again, the mobility separation shown on the y-axis displays two distinct peaks, the first at 23.49 $\mathrm{ms}$ and the second at $22.63 \mathrm{~ms}$; while the $\mathrm{m} / \mathrm{z}$ dimension displays a singular peak at $\mathrm{m} / \mathrm{z} 449$ corresponding to the $[\mathrm{M}+\mathrm{Na}]^{+}$complex. To our knowledge, this is the first report of isobaric carbohydrate separations by atmospheric pressure ion mobility. With an average measured resolving power of 97.8 , the resolution of these linkage isomers is 2.11 , well above the 1.5 necessary for baseline resolution. The measured ion neutral cross sections for these two sodium-disaccharide adducts were $164(\beta-[1-3])$ and $170 \AA^{2}(\beta-[1-6])$. Adducts of potassium for both $\beta$-D-GlcNAc-(1-6)-D-GalNAc-ol and $\beta$-D-GlcNAc-(1-3)-D-GalNAc-ol were also identified as shown in Figure 4. These low intensity contours are believed to be a result of trace levels of potassium found within the sample but do not greatly contribute to the overall measurement.

The experimentally determined ion-neutral collision cross sections for the $\mathrm{N}$-acetyl hexosamine alditols ranged from 161 to $170 \AA^{2}$. Given this wide array of cross sections it was possible to identify three different disaccharide isomers using AP-IM-TOF-MS (Figure 5). The 3-dimensional contours correspond to the following disaccharide isomers: $\beta$-D-GlcNAc-(1-6)-D-GalNAc-ol, $\beta$-D-GlcNAc-(1-
4)-D-GlcNAc-ol, and $\alpha$-D-GalNAc-(1-3)-D-GalNAc-ol. This spectrum again demonstrates the ability of AP-IMTOF-MS to separate and identify isobaric species from within simple carbohydrate mixtures. In this experiment, the two structures containing a GalNAc-ol residue were isolated from bovine submaxillary mucin, demonstrating the potential of these separations for analysis of glycoprotein-derived oligosaccharides.

Unfortunately, the ion gating mechanism of IMS results in duty cycles on the order of $1 \%$ throughout the experiment. Thus, the information gained through the union of ion mobility and mass spectrometry routinely requires a sacrifice in sensitivity. However, the addition of IMS to MS has also been shown to decrease chemical noise in the mass spectrometer. Based on the measured SNR (77-373) for the disaccharides the method detection limit (three times the standard deviation of the noise) for this class of compounds ranged from 24-117 pmol.

\section{Trisaccharide Separation}

To further explore the ability of AP-IM-TOFMS to distinguish between sodium adducted carbohydrates, a series of three trisaccharides were examined. The separation of and equimolar mixture $(50 \mathrm{pmol} / \mu \mathrm{L})$ of these three trisaccharides is shown in Figure 6. The reduced mobility values and ion-neutral collision cross sections 


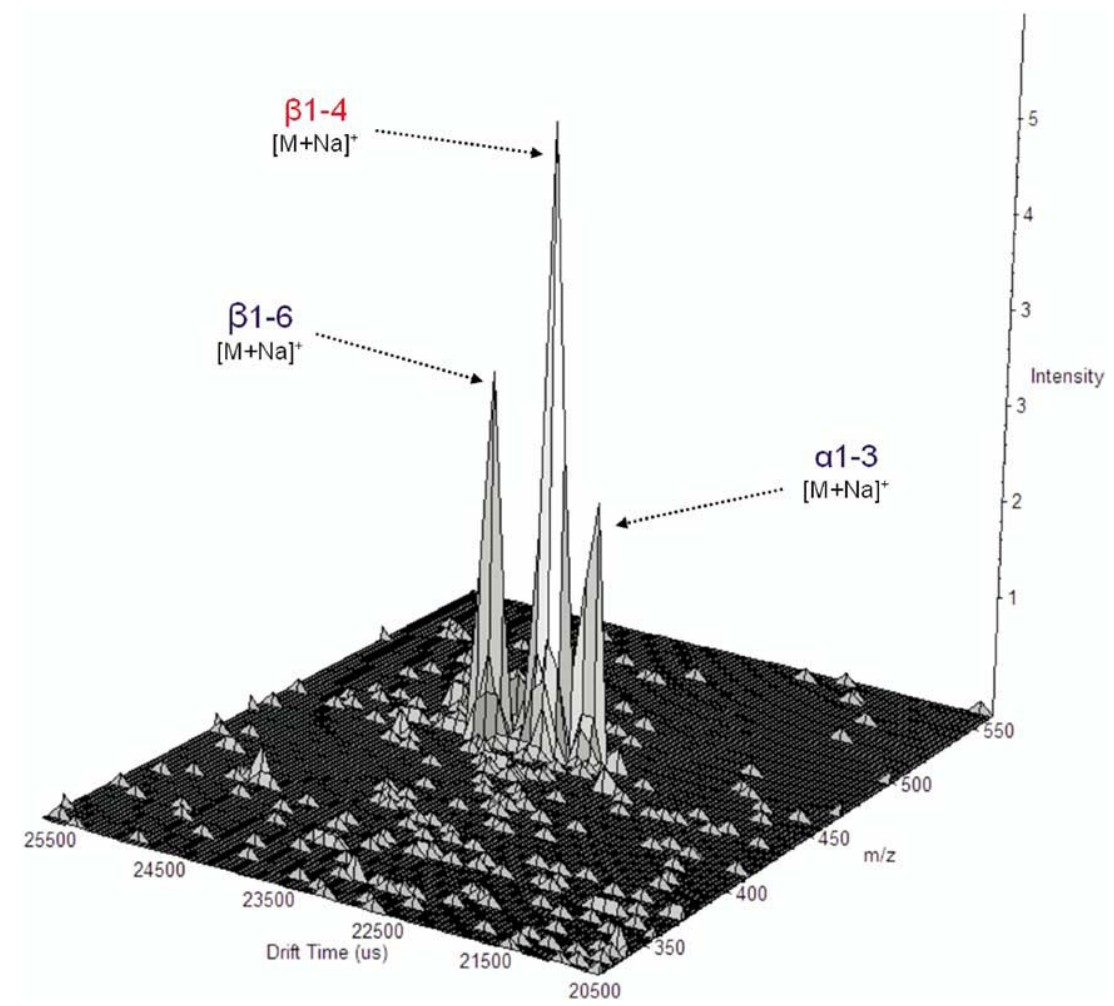

Figure 5. Separation of an equimolar mixture $(100 \mathrm{pmol} / \mu \mathrm{L})$ of three isobaric disaccharide sodium adducts. Listed in order of decreasing drift time: $\beta$-D-GlcNAc-(1-6)-D-GalNAc-ol, $\beta$-D-GlcNAc-(1-4)D-GlcNAc-ol, and $\alpha$-D-GalNAc-(1-3)-D-GalNAc-ol, with respective SNRs of 90, 162, and 67.

for the sodium adducted trisaccharides shown in Figure 6 are summarized in Table 1 . The contour data shown in Figure 6 represents the data from the sample mixture, with the 1D mass spectrum for this separation on the upper $\mathrm{x}$-axis and the $1 \mathrm{D}$ mobility separation on the y-axis. The outer $1 \mathrm{D}$ traces illustrate the mass and mobility data obtained for each trisaccharide standard.

Of these trisaccharides, melezitose, and raffinose are special instances in which the anomeric carbon of the terminal sugar is involved with inter-residue bonding. The non-reducing character of these trisaccharides makes chemical reduction unnecessary. As discussed in the previous section, sodium borohydride treatment of reducing disaccharides was conducted in order to eliminate the possibility of multiple conformations existing in solution phase. However, in the case of isomaltotriose, the third of the trisaccharides examined, this reduction was not performed. Despite the presence of a reducing terminal residue on isomaltotriose, only one stable ion population was observed indicating an absence of interconversion during the course of the experiment. It should be noted that while reduction was not performed and a single ion population was observed, this does not preclude the possibility of interconversion at the terminal anomeric carbon.

As with the disaccharides, no fragmentation was seen during the course of the ion mobility experiment. However, for the individual standards, most notably melezitose, comparatively small peaks are seen at $\mathrm{m} / \mathrm{z}$
355 and 371, which are also responsible for the ion mobility peak observed at $21.4 \mathrm{~ms}$ on the $\mathrm{y}$-axis trace of the melezitose standard. These ions appeared to be the result of impurities in solution and were clearly not the result of gas-phase fragmentation of parent ions. These traces were not seen during the mixture separation, perhaps because of charge competition and the comparatively large SNR of the major mixture constituents. Despite the presence of the impurities, it was possible to distinguish between three isomeric sodium adducts of the trisaccharides, melezitose, raffinose, and isomaltotriose. The average SNR for the trisaccharides standards examined was $272 \pm 30$ which corresponds to an estimated method detection limit of $17 \pm 2$ pmol.

\section{Conclusions}

Ion-neutral collision cross sections and separation characteristics were determined for the range of disaccharides isolated from bovine submaxillary mucin. These parameters were also reported for the trisaccharides melezitose, raffinose, and isomaltotriose. Using AP-IMTOF-MS it was possible to distinguish between three different isobaric species of both di- and trisaccharides. Further this technique demonstrated the ability to separate linkage isomers of disaccharides with greater than baseline resolution. Thus, it is clear that the current instrumental configuration allows for the unambiguous separation of simple carbohydrate mixtures. 


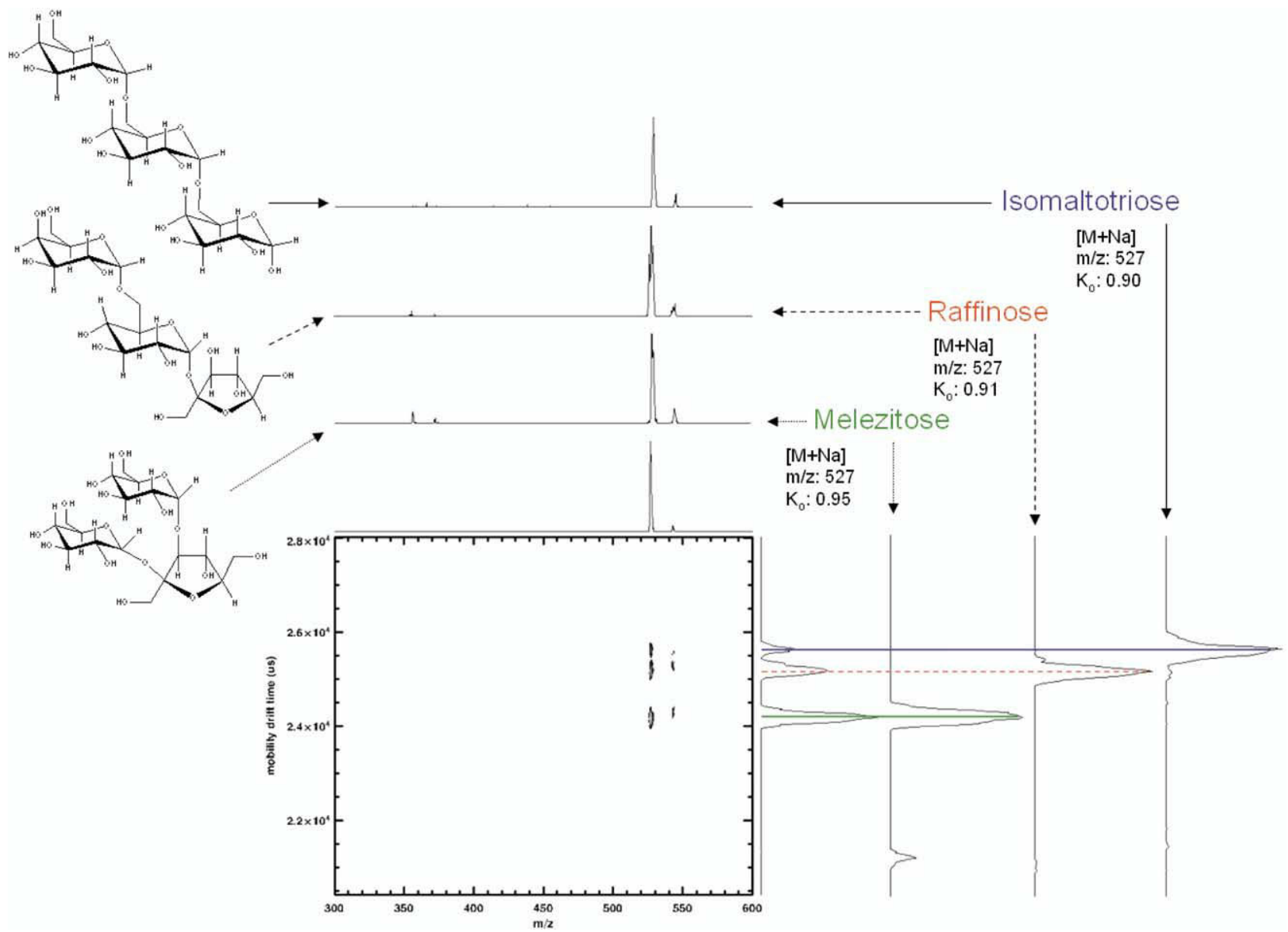

Figure 6. An ion mobility/mass spectral contour plot showing the equimolar $(50 \mathrm{pmol} / \mu \mathrm{L})$ mixture separation of the sodium adducts of melezitose, raffinose, and isomaltotriose. The most inner set of mass and mobility spectra represent the mixture separation, whereas the outer 1D traces correspond to the individual standards. The peaks within the mixture are correlated with the respective standard as illustrated by the horizontal lines extending to the outer ion mobility spectra. The SNRs of the standards melezitose, raffinose, and isomaltotriose were 294, 384, and 237, respectively.

The data obtained in this study demonstrate the utility of AP-IM-TOF-MS for the separation and identification of isobaric carbohydrate sodium adducts. While this technique has not reached the level necessary to supplant MS fragmentation studies as a primary means of carbohydrate identification it may be used conjointly and provide information in a dimension complementary to $\mathrm{m} / \mathrm{z}$ measurements. Moreover, in the future mobility drift gases of differing selectivity may be used to influence the order and degree of separation to provide a broader range of information regarding the gas-phase ion structures of metal coordinated carbohydrate ions prior to mass analysis [43, 44].

\section{Acknowledgments}

This work was supported by the Summer Research Experience program of the NSF Integrative Graduate Education and Research Training (IGERT) grant under NSF grant DGE-9972817 to Washington State University. BB acknowledges NSF grant CHE-0137986 for partial support.

\section{References}

1. Hakomori, S. Structure, organization, and function of glycosphingolipids in membrane. Curr. Opin. Hematol. Rev. 2003, 10(1), 16-24.

2. Soric., M.; Longo, A.; Garofalo, T.; Mattei, V.; Misasi, R.; Pavan, A. Role of GM3-enriched microdomains in signal transduction regulation in T lymphocytes. Glycoconj. J. 2004, 20(1), 63-70.

3. Fredman, P.; Hedberg, K.; Brezicka., T. Gangliosides as therapeutic targets for cancer. BioDrugs Rev. 2003, 17(3), 155-167.

4. Ramasamy, R.; Reese, R. T. A role for carbohydrate moieties in the immune response to malaria. J. Immunol. 1985, 134(3), 1952-1955.

5. Voet, D.; Voet, J. G. Biochemistry; 2nd ed; John Wiley \& Sons: New York, 1995; p 1361.

6. Kaufman, R. J.; Scheuner, D.; Schroeder, M.; Shen, X.; Lee, K.; Liu, C. Y.; Arnold, S. M. The unfolded protein response in nutrient sensing and differentiation. Nat. Rev. Mol. Cell Biol. 2002, 3, 411-421.

7. Varki, A.; Cummings, R.; Esko, J.; Freeze, H.; Hart, G.; Marth, J., Eds.; Essentials of Glycobiology; Cold Spring Harbor: New York, 1999; p 653.

8. Zaia, J. Mass spectrometry of oligosaccharides. Mass Spectrom. Rev. 2004, 23(3), 161-227. 
9. Angel, A. S.; Nilsson, B. Linkage positions in glycoconjugates by periodate oxidation and fast atom bombardment mass spectrometry. Methods Enzymol. 1990, 193, 587-607.

10. Pappas, R. S.; Sweetman, B.; Ray, S.; Hellerqvist, C. G. Monomer sequence determination of carbohydrates using fast-atom bombardment mass-spectrometry of periodate oxidized acetate ester derivatives. Carbohydr. Res. 1990, 197, 1-14.

11. Desaire, H.; Leary, J. A. Differentiation of diastereomeric $\mathrm{N}$-acetylhexosamine monosaccharides using ion trap tandem mass spectrometry. Anal. Chem. 1999, 71, 1997-2002.

12. Gaucher, S. P.; Leary, J. A. Determining anomericity of the glycosidic bond in $\mathrm{Zn}$ (II)-diethylenetriamine-disaccharide complexes using $\mathrm{MS}^{\mathrm{n}}$ in a quadrupole ion trap. J. Am. Soc. Mass Spectrom. 1999, 10, 269-272.

13. Zhang, H. L.; Reinhold, V. Composition to sequence: A novel computational approach to support $\mathrm{MS}^{\mathrm{n}}$ sequencing. Glycobiology 2003, 13, 81.

14. Itoh, S.; Kawasaki, N.; Ohta, M.; Hyuga, M.; Hyuga, S.; Hayakawa, T. Simultaneous microanalysis of N-linked oligosaccharides in a glycoprotein using microbore graphitized carbon column liquid chromatography-mass spectrometry. J. Chromatogr. A 2002, 968(1/2), 89-100.

15. Asbury, G. R.; Klasmeier, J.; Hill, H. H., Jr. Analysis of explosives using electrospray ionization/ion mobility spectrometry (ESI/IMS). Talanta 2000, 50(6), 1291-1298.

16. Eiceman, G. A.; Karpas, Z. Ion Mobility Spectrometry; CRC Press: Boca Raton, FL, 1994; pp 192-197.

17. Steiner, W. E.; Clowers, B. H.; Haigh, P. E.; Hill, H. H., Jr. Secondary ionization of chemical warfare agent simulants: Atmospheric pressure ion mobility time-of-flight mass spectrometry. Anal. Chem. 2003, 75(22), 6068-6076.

18. Buxton, T. L.; Harrington, P. de B. Trace explosive detection in aqueous samples by solid-phase extraction ion mobility spectrometry (SPE-IMS). Appl. Spectrosc. 2003, 57(2), 223-232.

19. Matz L. M; Hill, H. H., Jr. Evaluation of opiate separation by high-resolution electrospray ionization-ion mobility spectrometry/mass spectrometry. Anal. Chem. 2001, 73(8), 1664-1669.

20. Wu, C.; Siems, W. F.; Asbury, G. Reid; Hill, H. H., Jr. Electrospray ionization high-resolution ion mobility spectrometry-mass spectrometry. Anal. Chem. 1998, 70(23), 4929-4938.

21. Liu, Y.; Valentine, S. J.; Counterman, A. E.; Hoaglund, C. S.; Clemmer, D. E. Injected-ion mobility analysis of biomolecules. Anal. Chem. 1997, 69, 728A.

22. Dugourd, P.; Hudgins, R. R.; Clemmer, D. E.; Jarrold, M. F. High resolution ion mobility measurments. Rev. Sci. Instrum. 1997,68(2), 1122-1129.

23. Borsdorf, H.; Nazarov, E. G.; Eiceman, G. A. Atmospheric pressure ionization and gas-phase ion mobility studies of isomeric dihalogenated benzenes using different ionization techniques. Int. J. Mass Spectrom. 2004, 232(2), 117-126.

24. Wu, C.; Siems, W. F.; Klasmeier, J.; Hill, H. H., Jr. Separation of isomeric peptides using electrospray ionization/high-resolution ion mobility spectrometry. Anal. Chem. 2000, 72(2), 391-395.

25. Srebalus-Barnes, C. A.; Hilderbrand, A. E.; Valentine, S. J.; Clemmer, D. E. Resolving isomeric peptide mixtures: A combined HPLC/ion mobility-TOFMS analysis of a 4000-component combinatorial library. Anal. Chem. 2002, 74(1), 26-36.

26. Lee, S.; Wyttenbach, T.; Bowers, M. T. Gas-phase structures of sodiated oligosaccharides by ion mobility/ion chromatography methods. Int. J. Mass Spectrom. Ion Processes 1997, 167/168 605-614.

27. Liu, Y.; Clemmer, D. E. Characterizing oligosaccharides using injected-ion mobility/mass spectrometry. Anal. Chem. 1997, $69,2504-2509$.
28. Lee, D.-S.; Wu, C.; Hill, H. H., Jr. Detection of carbohydrates by electrospray ionization-ion mobility spectrometry following microbore high-performance liquid chromatography. J. Chromatog. A. 1998, 822, 1-9.

29. Leavell, M. D.; Gaucher, S. P.; Leary, J. A.; Taraszka, J. A.; Clemmer, D. E. Conformational studies of Zn-ligand-hexose diastereomers using ion mobility measurements and density functional theory calculations. J. Am. Soc. Mass Spectrom. 2002, 13(3), 284-293.

30. Gabryelski, W; Froese, K. L. Rapid and sensitive differentiation of anomers, linkage, and position isomers of disaccharides using high-field asymmetric waveform ion mobility spectrometry (FAIMS). J. Am. Soc. Mass Spectrom. 2003, 14(3), 265-277.

31. Martensson, S.; Levery, S. B.; Fang, T. T.; Bendiak, B. Neutral core oligosaccharides of bovine submaxillary mucin. Use of lead tetra-acetate in the cold for establishing branch positions. Eur. J. Biochem. 1998, 258(2), 603-622.

32. Steiner, W. E.; Clowers, B. H.; Fuhrer, K.; Gonin, M.; Matz, L. M.; Siems, W. F.; Schultz, A. J.; Hill, H. H., Jr. Electrospray ionization with ambient pressure ion mobility separation and mass analysis by orthogonal time-of-flight mass spectrometry. Rapid Communi. Mass Spectrom. 2001, 15(23), 2221-2226.

33. Hill, H. H., Jr.; Hill, C. H.; Asbury, G. R.; Wu, C.; Matz, L. M.; Ichiye, T. Charge location on gas-phase peptides. Int. J. Mass Spectrom. 2002, 219(1), 23-37.

34. Wyttenbach, T.; Bowers, M. T. Gas-phase conformations: The ion mobility/ion chromatography method. Topics in Current Chemistry. Modern Mass Spectrom. 2003, 225, 207-232.

35. Siems, W. F.; Wu, C; Tarver, E. E.; Hill, H. H., Jr.; Larsen, P. R.; McMinn, D. G. Measuring the resolving power of ion mobility spectrometers. Anal. Chem. 1994, 66(23), 4195-201.

36. Harris, D. C. Quantitative Chemical Analysis, 5th ed.; Freeman and Co.: New York, pp 654-655.

37. Revercomb, H. E.; Mason, E. A. Theory of plasma chromatography/gaseous electrophoresis. Anal. Chem. Rev. 1975, 47(7), 970-983.

38. Shvartsburg, A. A.; Jarrold, M. F. An exact hard-spheres scattering model for the mobilities of polyatomic ions. Chem. Phys. Lett. 1996, 261, 86-91.

39. Mesleh, M. F.; Hunter, J. M.; Shvatrsburg, A. A.; Schatz, G. C.; Jarrold, M. F. Structural information from ion mobility measurements: Effects of the long-range potential. J. Phys. Chem. 1996, 100, 16082-16086.

40. Wyttenback, T.; Batka, J. J., Jr.; Gidden, J.; Bowers, M. T. Host/guest conformation of biological systems: Valinomycin/ alkali ions. Int. J. Mass Spectrom. 1999, 193, 143-152.

41. Stone, E. G.; Gillig, K. J.; Ruotolo, B. T.; Russell, D. H. Optimization of a matrix-assisted laser desorption ionizationion mobility-surface-induced dissociation-orthogonal-time-offlight mass spectrometer: Simultaneous acquisition of multiple correlated MS1 and MS2 spectra. Int. J. Mass Spectrom. 2001, 212(1/3), 519-533.

42. Cerda, B. A.; Wesdemiotis, C. Thermochemistry and structures of $\mathrm{Na}+$ coordinated mono- and disaccharide stereoisomers. Int. J. Mass Spectrom. 1999, 189(2/3), 189-204.

43. Asbury, G. R.; Hill, H. H., Jr. Using different drift gases to change separation factors $(\alpha)$ in ion mobility spectrometry. Anal. Chem. 2000, 72(3), 580-584.

44. Matz, L. M.; Hill, H. H., Jr. Beegle, L. W.; Kanik, I. Investigation of drift gas selectivity in high resolution ion mobility spectrometry with mass spectrometry detection. J. Am. Soc. Mass Spectrom. 2002, 13(4), 300-307. 